4 CELMA, S., SABADELL, J., and MARTINEZ, P.: 'Universal filter using unity-gain cells', Electron. Lett., 1995, 31, pp. 1817-1818

5 BRUUN, E.: 'Constant-bandwidth current mode operational amplifier', Electron. Lett., 1991, 27, pp. 1673-1674

\section{Insensitive current/voltage-mode filters using FTFNs}

\author{
Shen-Iuan Liu and Jiin-Long Lee
}

\begin{abstract}
Indexing terms: Current-mode circuits, Active filters, Analogue circuits

A new configuration for realising insensitive current/voltagemode filters using two four-terminal floating nullors (FTFNs) is presented. It can provide the current/voltage-mode filters with reduced sensitivities. Moreover, the current-mode filters can have high output impedance and only grounded capacitors. The proposed universal voltage-mode filter with dual outputs can realise lowpass, bandpass, highpass, notch and allpass filtering functions. Simulation results are given to verify the theoretical analysis.
\end{abstract}

Introduction: Current-mode circuits have been receiving significant attention besause they have the potential advantages of accuracy and wide bandwidth over their voltage-mode counterparts [1]. To be cascaded without additional current buffers, it is desirable for current-mode filters to have high output impedance. Therefore, several cascadable current-mode filters [2 - 6] using second generation current conveyors (CCIIs) and single four terminal floating nullor (FTFN) [7, 8] have been developed. Recently, a cascadable current-mode filter [6] using single FTFN was presented. However, its active sensitivities were large and it requires a floating capacitor. In this Letter, a new configuration is presented to provide the current/voltage-mode filters with reduced sensitivities. Simulation results are given to verify the theoretical analysis.
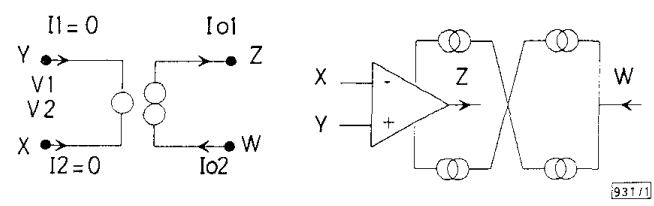

Fig. 1 Nullor model of FTFN

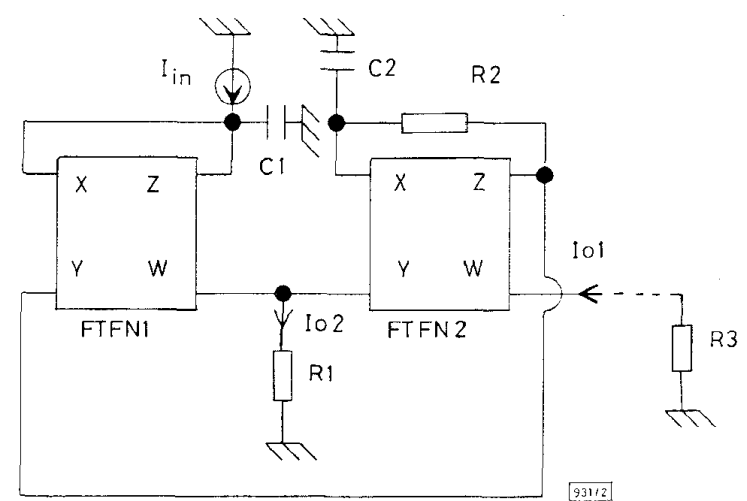

Fig. 2 Proposed current-mode filter with high output impedance

Circuit description: An FTFN is equivalent to an ideal nullor [68]. The port relations of an FTFN, shown in Fig. 1, can be characterised as $I_{1}=I_{2}=0, V_{1}=V_{2}$ and $I_{o 1}=I_{o 2}$. Considering the proposed current-mode configuration in Fig. 2 , its transfer functions can be derived as

$$
\frac{I_{o 1}}{I_{\text {in }}}=\frac{s C_{2} R_{1}}{1+s C_{1} R_{1}+s^{2} C_{1} C_{2} R_{1} R_{2}}
$$

$$
\frac{I_{o 2}}{I_{i n}}=\frac{1}{1+s C_{1} R_{1}+s^{2} C_{1} C_{2} R_{1} R_{2}}
$$

It can simultaneously realise a bandpass filter and a lowpass filter. The natural frequency and quality factor of this filter can be expressed as

$$
\omega_{o}=\frac{1}{\sqrt{C_{1} C_{2} R_{1} R_{2}}} \text { and } Q=\sqrt{\frac{R_{2} C_{2}}{R_{1} C_{1}}}
$$

taking into account the nonideal FTFN, i.e. $V_{2}=\alpha V_{1}$ and $I_{i 2}=$ $\beta I_{o !}$ where $\alpha=1-\varepsilon_{1}$ and $\varepsilon_{1}\left(\varepsilon_{1} \ll 1\right)$ denotes the voltage tracking error of an FTFN; and $\beta=1-\varepsilon_{2}$ and $\varepsilon_{2}\left(\varepsilon_{2} \ll 1\right)$ is the current tracking error. Since the current output of the FTFN2 in Fig. 2 is directly connected to the load $\left(R_{3}\right)$, the resultant current-mode filters will be insensitive to the current tracking error of the FTFN2 except for current gain. The transfer function $I_{o 1} / I_{i n}$ in Fig. 2 can be rewritten as

$$
\frac{I_{o 1}}{I_{i n}}=\frac{\alpha_{1} \beta_{2} s C_{2} R_{1}}{\alpha_{1} \alpha_{2} / \beta_{1}+s C_{1} R_{1}+s^{2} C_{1} C_{2} R_{1} R_{2}}
$$

It can be found that the passive and active sensitivities of this filter are

$$
\begin{aligned}
& S_{C_{1}}^{\omega_{0}}=S_{C_{2}}^{\omega_{0}}=S_{R_{1}}^{\omega_{0}}=S_{R_{2}}^{\omega_{0}}=-0.5 \\
& S_{C_{2}}^{Q}=-S_{C_{1}}^{Q}=-S_{R_{1}}^{Q}=S_{R_{2}}^{Q}=0.5 \\
& S_{\alpha_{1}}^{\omega_{0}}=S_{\alpha_{2}}^{\omega_{0}}=-S_{\beta_{1}}^{\omega_{0}}=S_{\alpha_{1}}^{Q}=S_{\alpha_{2}}^{Q}=-S_{\beta_{1}}^{Q}=0.5 \\
& S_{\beta_{2}}^{\omega_{0}}=S_{\beta_{2}}^{Q}=0
\end{aligned}
$$

Thus, the passive and active sensitivities of this filter are all low. Moreover, this filter can provide a high impedance output and it contains only grounded capacitors.

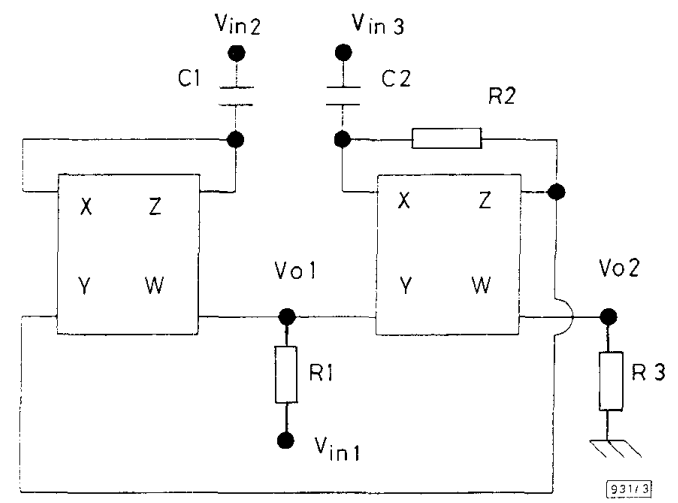

Fig. 3 Voltage-mode universal filter

The proposed voltage-mode universal filter is shown in Fig. 3 . Its transfer function can be expressed as

$$
\begin{gathered}
V_{o 1}=\frac{s^{2} C_{1} C_{2} R_{1} R_{2} V_{i n 3}+s C_{1} R_{1} V_{i n 2}+V_{i n 1}}{1+s C_{1} R_{1}+s^{2} C_{1} C_{2} R_{1} R_{2}} \\
V_{o 2}=s C_{2} R_{3}\left(V_{o 1}-V_{i n 3}\right)
\end{gathered}
$$

If $V_{i n 2}=V_{i n 3}=0$, a lowpass function and a bandpass function can be obtained at $V_{o 1}$ and $V_{o 2}$, respectively. If $V_{i n 1}=V_{i n 3}=0$, a bandpass function and a highpass function can be achieved at $V_{o 1}$ and $V_{i 2}$, respectively. Moreover, if $V_{i l 2}=0$ and $V_{i n 1}=V_{i n 3}$, a notch function and a bandpass function can be obtained at $V_{o 1}$ and $V_{02}$. To realise the allpass filter, input signals must have the following relations: $V_{i n 1}=-V_{i r 2}=V_{i n}$. Its natural frequency and quality factor are given in eqn. 3. Its passive and active sensitivities are also low.

Simulation results: To verify the theoretical analysis. the proposed current/voltage-mode filters have been simulated, based on the advanced PSPICE macromodels [9]. The FTFN [8] consists of the AD704 [10] Op Amp, Q2N2222A NPN and Q2N2907A PNP transistors. As an example, the simulation results of the proposed current-mode bandpass filter in Fig. 2 were presented with $C_{1}=C_{2}$ $=10 \mathrm{nF}, R_{1}=1 \mathrm{k} \Omega$, and $R_{2}=R_{3}=25 \mathrm{k} \Omega$. Fig. 3 shows the simulation results of the second order bandpass filter. Simulation results conform with the results of the theoretical analysis. Other proposed filters have also been verified as working properly by PSPICE macromodels. 


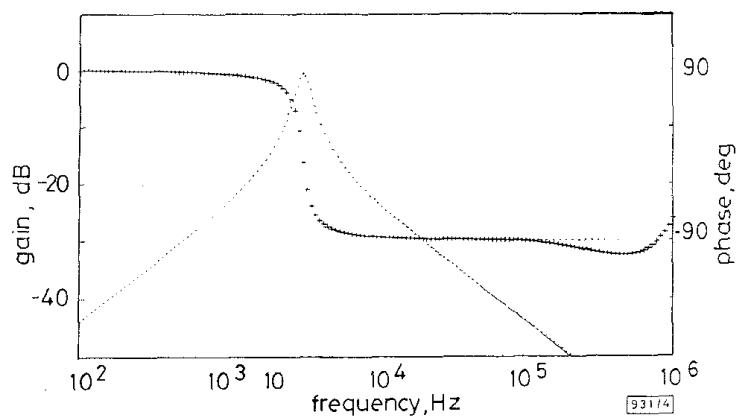

Fig. 4 Comparisons between theoretical and simulated results for bandpass filter in Fig. 2 with $C_{1}=C_{2}=10 \mathrm{nF}, R_{1}=1 \mathrm{k} \Omega$ and $R_{2}=R_{3}=$

Theoretical: - gain, --- phase
Simulated: $\cdots . .$. gain, +++ phase

Conclusions: The proposed circuits have been thoroughly verified by using the PSPICE macromodel simulations. This configuration can provide the current/voltage-mode filters with reduced sensitivities. Moreover, the current-mode bandpass can have high output impedance and only grounded capacitors. The voltage-mode filter can be a universal filter. The proposed circuits are expected to be useful in analogue filtering applications.

\section{(C) IEE 1996}

Electronics Letters Online No: 19960729

16 April 1996

Shen-Iuan Liu and Jiin-Long Lee (Department of Electrical Engineering, National Taiwan University, Taipei, Taiwan 10664, Republic of China)

\section{References}

1 WILSON, B.: 'Recent developments in current conveyors and currentmode circuits', IEE Proc. G, Circuits Devices Syst., 1990, 137, (2), pp. $63-77$

2 LIU, S.I., TSAO, H.W, and WU, J.: 'Cascadable current-mode single CCII biquads', Electron. Lett., 1990, 26, pp. 2005-2006

3 CHANG, C.M.: 'Current-mode allpass/notch and bandpass filter using single CCII', Electron. Lett., 1991, 27, pp. 1812-1813

4 NANDI, R.: 'Novel current-mode all-pass phase shifter using a current conveyor', IEEE Trans. Instrum. Meas., 1992, IM-41, pp. $553-555$

5 HIGASHIMURA, M.: 'Realisation of current-mode transfer function using four-terminal floating nullor', Electron. Lett., 1991, 27, pp. $170-171$

6 LIU, S.I.: 'Cascadable current-mode filters using single FTFN', Electron. Lett., 1995, 31, pp. 1965-1966

7 HUIJSING, J.H.: 'Operational floating amplifier', IEE Proc. G, Circuits Devices Syst., 1990, 137, pp. 131-136

8 SENANI, R: 'On equivalent forms of single op-amp sinusoidal RC oscillators', IEEE Trans. Circuits Syst. I, Fundam. Theory Appl., 1994, CAS-41, pp. 617-624

9 MicroSim Corp., Irvine, California, 1993

10 Analog Devices, Norwood MA, 1990

\section{Linearly constrained block adaptive filtering algorithm with optimum convergence factors}

T. Demeechai

Indexing terms: Adaptive filters, Array signal processing

A new linearly constrained adaptive filtering algorithm, the linearly constrained optimum block adaptive (LCOBA) algorithm, is presented. The LCOBA algorithm processes data in blocks and uses variable convergence factors which are optimised in a least squares sense. It is superior to Frost's linearly constrained least mean square algorithm at achieving the conflicting goals of fast convergence with little steady-state error. In addition, its computational requirement generally tends to be smaller than that of the Frost algorithm, as the block length is increased.
Introduction: Linearly constrained adaptive filtering is an important tool in array processing problems. The most widely used algorithm, owing to its simplicity, efficiency and robustness, is the linearly constrained least mean square algorithm proposed by Frost [1]. The convergence properties of the Frost algorithm critically depend on a fixed parameter known as the convergence factor. A relatively large convergence factor may result in fast convergence but large steady-state error. The proper value of the convergence factor must be selected to balance the conflicting goals of fast convergence and small steady-state error. This usually requires prior information about the adaptive filter input process, as well as trial and error.

This Letter presents a new block gradient algorithm for linearly constrained adaptive filtering which uses convergence factors optimised in a least squares sense for each update of the filter weight vector: the linearly constrained optimum block adaptive (LCOBA) algorithm. The expression for the optimum convergence factors is derived simply, and in a similar way to that in [2]. In the LCOBA algorithm, the blocks of processed signals are disjointed: therefore, the computational requirement of the new algorithm tends generally to be smaller than that of the Frost algorithm, as the block length is increased. Computer simulations are also conducted for evaluating the convergence properties of the LCOBA algorithm. The results show that the new algorithm is superior to the Frost algorithm at achieving the conflicting goals of fast convergence with little steady-state error.

The LCOBA algorithm: A linearly constrained adaptive filter may be considered as an FIR filter with an adaptive process, for minimising the output mean square error, that recursively adjusts the filter weight vector $W$ which is constrained by an equation such as: $C^{H} W=F$, where the superscript $(\cdot)^{H}$ denotes complex conjugate and transpose. The weight vector has dimensions $N \times 1$, where $N$ is the number of filter taps. The constraint matrix $C$ has dimensions $N \times K$, where $K$ is the number of constraints. The idea behind the LCOBA algorithm is similar to that behind the Frost algorithm, since the LCOBA algorithm uses a negative gradient, orthogonalised to all of the constraint matrix column vectors, multiplied by a convergence factor and then added with a correction term for each recursion. However, the LCOBA algorithm works with disjointed blocks of data and uses variable convergence factors optimised in a least squares sense. In LCOBA algorithms with block length $L$, the error signal matrix for each data block is defined by

$$
E_{j}=D_{j}-W_{j}^{H} X_{j}
$$

where the subscript $D$ and $X$ are, respectively, the block index, the $1 \times L$ desired signal matrix and the $N \times L$ input signal matrix. It can be shown that half the negative gradient for the $j$ th block sum square error $E_{j} E_{j}^{H}$ equals $X_{j} E_{j}^{H}$. Therefore, after being initialised by $W_{0}=C\left(C^{H} C\right)^{-1} F$, the LCOBA algorithm is based on the recursion

$$
\begin{aligned}
W_{j+1}=W_{j} & +\mu_{j}\left(I-C\left(C^{H} C\right)^{-1} C^{H}\right) X_{j} E_{j}^{H} \\
& +C\left(C^{H} C\right)^{-1}\left(F-C^{H} W_{j}\right)
\end{aligned}
$$

where $I$ is the identity matrix and $\mu_{j}$ is a real number representing the optimum convergence factor for $j$ th recursion. This convergence factor is optimal in the sense that it minimises the a posteriori block sum square error $\xi_{j} \xi_{j}^{H}$, where

$$
\xi_{j}=D_{j}-W_{j+1}^{H} X_{j}
$$

Owing to the fact that $\xi_{j} \xi_{j}{ }^{H}$ is a quadratic function of the filter weight vector, it can be shown (from eqns. 2 and 3 ) that $\xi_{j} \xi_{j}{ }^{H}$ may be expressed by a parabolic function of the convergence factor, and the optimum convergence factor is

$$
\mu_{j}=\frac{Q_{j}^{H} X_{j} E_{j}^{H I}}{Q_{j}^{H} X_{j} X_{j}^{H} Q_{j}}
$$

where $Q_{j}=\left(I-C\left(C^{H} C\right)^{-1} C^{H}\right) X_{j} E_{j}^{H}$

Computer simulations: To illustrate the performance of the LCOBA algorithm, computer simulations on minimum variance filtering similar to those in [3] have been conducted. The performance measure is the normalised error energy $(N E E)$ defined by

$$
N E E=\frac{\left(W-W_{o p}\right)^{H}\left(W-W_{o p}\right)}{W_{o p}^{H} W_{o p}}
$$

